\title{
PMR-relevante Zeitschriften - Eine Liste wissenschaft- licher Zeitschriften für Physikalische Medizin und Rehabilitation sowie verwandter Fachbereiche
}

\section{S. Piek ${ }^{1}$ \\ P. Kröling' ${ }^{1}$ \\ K. Ammer ${ }^{3}$ \\ G. Stucki ${ }^{2}$}

\author{
PMR-Relevant Journals - A List of Journals for Physical Medicine \\ and Rehabilitation and Related Fields
}

\section{Zusammenfassung}

Ziel dieses Beitrags ist es, dem Leser einen Überblick über relevante Zeitschriften aus dem Bereich der Physikalischen Medizin und Rehabilitation (PMR) zu ermöglichen, die englisch- oder deutschsprachig publiziert und über Internet zugängig sind. Durch die Einbeziehung des Impact Factors sowie weiterer Merkmale (Herausgeber, Organisation etc.) soll die Liste darüber hinaus ermöglichen, im Sinne der EBM das wissenschaftliche Gewicht der jeweiligen Zeitschrift auf einen Blick grob abzuschätzen. Charakteristisch für die PMR sind zahlreiche Überschneidungen mit anderen Fachgebieten wie Orthopädie, Neurologie, Rheumatologie, Geriatrie, Epidemiologie und Public Health. Deshalb wurde die Auflistung auch um diejenigen Zeitschriften aus verwandten Fachgebieten erweitert, in denen häufig auch Beiträge zu PMR-relevanten Themen erscheinen. 132 Titel wurden aus einer Gesamtliste mit rund 6000 Titeln selektiert, die faktisch oder potenziell PMR-relevant sind. Die hier vorgestellte Auswahlliste wird auch auf der Website der Deutsche Gesellschaft für Physikalische Medizin und Rehabilitation* zur Verfügung gestellt (DGPMR: www.dgpmr.de).

\section{Schlüisselwörter}

Zeitschriftenliste · Physikalische Medizin · Rehabilitation • Physiotherapie $\cdot$ Impact Factor

\section{Abstract}

This article aims to provide the reader with a list of journals in english or german language, that are relevant to Physical Medicine and Rehabilitation (PMR) and accessible by Internet. Impact Factor and other characteristics such as the editor and organisation have been included. The list will enable the reader to make an initial evaluation of the journals scientific value in terms of the principles of Evidence Based Medicine. Typical for PMR is the overlapping with other medical fields such as orthopaedics, neurology, rheumatology, geriatrics, epidemiology and public health. Journals from related medical fields, that frequently publish articles of relevance to PMR, are therefore also included. The list of 132 titles presented in this article has been selected from a comprehensive list of approximately 6000 titles that are potentially relevant to PMR. The presented list will also be available on the Website of the German Society of Physical Medicine and Rehabilitation (DGPMR: www.dgpmr.de).

\section{Key words}

Journal list · physical medicine $\cdot$ rehabilitation · physiotherapy · impact factor

Institutsangaben

${ }^{1}$ Institut f. Med. Balneologie und Klimatologie der Ludwig-Maximilians-Universität München

(Vorstand: Prof. Dr. med. Gerold Stucki)

${ }^{2}$ Klinikum der Ludwig-Maximilians-Universität München, Klinik und Poliklinik für Physikalische Medizin

und Rehabilitation Großhadern (Direktor: Prof. Dr. med. Gerold Stucki)

${ }^{3}$ Ludwig Boltzmann Forschungsstelle für Physikalische Diagnostik, Wien

Hinweis

* Die Auswahlliste von PMR-relevanten Journals (Tab. 2) wurde in Abstimmung mit der

Deutschen (DGPMR) und Österreichischen (ÖGPMR) Gesellschaft für Physikalische Medizin erstellt

Korrespondenzadresse

Prof. Dr. med. Peter Kröling · Institut für Medizinische Balneologie und Klimatologie

der Ludwig-Maximilians-Universität München · Marchioninistraße 17 · 81377 München

E-mail: kroeling@imbk.med.uni-muenchen.de

Eingegangen: 24. Februar 2004 · Angenommen: 27. Juli 2004

Bibliografie

Phys Med Rehab Kuror 2004; 14: 254 - 262 @ Georg Thieme Verlag KG Stuttgart · New York

DOI 10.1055/s-2004-828300

ISSN 0940-6689 


\section{Einführung}

An die Deutsche Gesellschaft für Physikalische Medizin und Rehabilitation e.V. (DGPMR) ist verschiedentlich der Wunsch herangetragen worden, eine Liste wissenschaftlich fundierter Zeitschriften für das Fachgebiet Physikalische Medizin und Rehabilitation (PMR) zu erstellen. Zu den Interessenten gehören einerseits wissenschaftlich arbeitende Kollegen und Medizinische Fakultäten, andererseits auch praktisch im Fachbereich tätige Ärzte, Physiotherapeuten und Lehrkräfte, die sich im Rahmen des ständig wachsenden Bedarfs an evidenzbasierter Literatur orientieren müssen. Weiterhin dient der Nachweis der Existenz einer qualitativ und quantitativ beachtlichen Fachliteratur auch zur Behauptung gegenüber anderen Fachbereichen, als Grundlage gesundheitspolitischer Entscheidungen und nicht zuletzt zum Selbstverständnis der PMR.

In den letzten Jahren sind die Möglichkeiten, sich über wissenschaftliche Erkenntnisse zu informieren, durch Internet und Datenbanken erheblich gewachsen. Praktisch alle relevanten PMRZeitschriften verfügen mittlerweile über eine eigene Internetseite (Website), die zumindest den Zugriff auf Titel und Abstracts, wenn nicht gar auf den vollen Text gestattet.

Ziel dieses Beitrags ist es zunächst, dem Leser einen Überblick über PMR-relevante Zeitschriften zu ermöglichen, die englischoder deutschsprachig publiziert und über Internet zugängig sind. Durch die Einbeziehung des Impact Factors sowie weiterer Merkmale (Herausgeber, Organisation etc.) soll die Liste darüber hinaus ermöglichen, im Sinne der EBM das wissenschaftliche Gewicht der jeweiligen Zeitschrift auf einen Blick grob abzuschätzen. Charakteristisch für die PMR sind zahlreiche Überschneidungen mit anderen Fachgebieten wie Orthopädie, Neurologie, Rheumatologie, Geriatrie, Epidemiologie und Public Health. Deshalb wurde die Auflistung auch um diejenigen Zeitschriften aus verwandten Fachgebieten erweitert, in denen häufig auch Beiträge zu PMR-relevanten Themen erscheinen.

\section{Methodik}

Es wurde zunächst im Rahmen einer umfangreichen Recherche eine Gesamtliste von rund 6000 Zeitschriftentiteln erstellt. Hieraus wurden 132 Titel für die unten stehende Tab. 2 ausgewählt.

Der vom Institut for Scientific Information (ISI) in Philadelphia (USA) veröffentlichte Journal Citation Report (JCR) [1] enthält alle Zeitschriften, welche über eine Impact Factor (IF) verfügen. Die PMR-relevanten Titel wurden in die Gesamtliste aufgenommen. In den Datenbanken CINAHL und MEDLINE wurden zuzüglich ebenfalls PMR-relevante Titel erfasst. Außerdem erfolgte eine Onlinerecherche über die Suchmaschine Google, jeweils kombiniert mit dem Begriff Journal oder Zeitschrift.

Die jeweiligen Suchstrategien wurden mit Hilfe von nachfolgenden Suchbegriffen ausgeführt:

\section{A) Suchbegriffe für Kernbereiche:}

- Physikalische Therapie/Medizin, physical therapy/medicine,

- rehabilitati*, krankengymnast*, physiotherap*,
- electrotherap*, elektrotherap*, electrical stimulation,

- thermotherap*, therapeutic heat, cryotherap*, therm* medicine/Medizin,

- ergotherap*, occupational therapy/medicine,

- Kurortmedizin, spa (treatment/medicine/therapy),

- balneolog*, balneotherap*,

- Medizinische Klimatologie, biometeorolog*, (bio)climatolog*,

- Manuelle Therapie/Medizin, manual therapy/medicine,

- massage, lympholog*.

B) Suchbegriffe für fachverwandte Gebiete:

- orthopäd*, orthop(a)edi*,

- biomechan*, sportmed* sport*, exercise,

- Schmerz, pain, neurolog*heumatol*,

- gerontolog*, geriatr*, aging,

- Gesundheitswesen, public health, epidemiolog*.

Die auf diese Art erfassten Titel wurden in neun Themen aufgeteilt, wovon die ersten 4 eher zu den Kernbereichen, die übrigen eher zu den fachverwandten Bereichen zählen (Anmerkung: für eine eigene Rubrik „Elektrotherapie“ wurden keine relevanten Titel gefunden):

1. Physikalische Medizin, Physikalische Therapie, Rehabilitation,

2. Physiotherapie („Krankengymnastik“), Ergotherapie (= Occupational Therapy),

3. Manuelle Therapie, Massage, Lymphologie,

4. Kurortmedizin, Balneotherapie, Thermotherapie, Med. Klimatologie,

5. Orthopädie, Rheumatologie,

6. Neurologie, Schmerz,

7. Sportmedizin, exercise,

8. Geriatrie, Gerontologie, Aging,

9. Epidemiologie, Public Health.

Innerhalb der jeweiligen Themenbereiche wurden die Titel nach der Höhe des vorhandenen Impact Factors aufgelistet, gefolgt von einer alphabetischen Reihenfolge der Titel ohne Impact Factor. Neben dem vollständigen Titel, dem Kurztitel und dem Impact Factor wurde auch Verlag, Herausgeber, Organ und die Websiteadresse angegeben. Die zweite Spalte enthält den Kurztitel (Short Titles) der jeweiligen Zeitschrift. Sie wurden in Großbuchstaben so übernommen, wie sie vom Institute for Scientific Information (ISI) in Philadelphia (USA) verwendet werden [1]. Bei Titel ohne Impact Factor wurden Abkürzungen anhand von Informationen auf den Websites der Zeitschriften verwendet (keine Großbuchstaben).

In der dritten Spalte befindet sich der Impact Factor für das Jahr 2002, herausgegeben von der ISI und aufgeführt im Journal Citation Report (JCR) [1]. Falls kein IF für 2002 vorlag, wurde das letzte Jahr mit IF separat vermerkt. Der IF 2002 gibt die durchschnittliche Häufigkeit an, mit der die in den Jahren 2000 und 2001 in dieser Zeitschrift publizierten Arbeiten in anderen wissenschaftlichen Publikationen des JCR zitiert wurden. Sie errechnet sich aus den Zitaten im Jahr 2002 von Artikeln aus 2000 und 2001, dividiert durch die Anzahl der wissenschaftlichen Beiträge aus den Jahren 2000 und 2001 (Schreiber 2002 [2]; Bowman 1998 [3]). 
Tab. 1 Aufteilung der gefundenen Zeitschriften (Gesamtliste) nach Fachbereichen und Sprache

\begin{tabular}{|c|c|c|c|}
\hline Themenbereich & $\begin{array}{l}\text { Titel } \\
\text { insgesamt }\end{array}$ & $\begin{array}{l}\text { englisch- } \\
\text { sprachige } \\
\text { Titel }\end{array}$ & $\begin{array}{l}\text { deutsch- } \\
\text { sprachige } \\
\text { Titel }\end{array}$ \\
\hline $\begin{array}{l}\text { Physikalische Medizin, Physikalische } \\
\text { Therapie, Rehabilitation }\end{array}$ & 679 & 427 & 33 \\
\hline $\begin{array}{l}\text { Physiotherapie („Krankengymnastik“), } \\
\text { Ergotherapie (= Occupational Ther- } \\
\text { apy) }\end{array}$ & 186 & 106 & 14 \\
\hline $\begin{array}{l}\text { Manuelle Therapie, Massage, } \\
\text { Lymphologie }\end{array}$ & 85 & 41 & 7 \\
\hline $\begin{array}{l}\text { Kurortmedizin, Balneotherapie, } \\
\text { Thermotherapie, Med. Klimatologie }\end{array}$ & 165 & 58 & 48 \\
\hline Orthopädie, Rheumatologie & 795 & 409 & 42 \\
\hline Neurologie, Schmerz & 1736 & 1237 & 64 \\
\hline Sportmedizin, Exercise & 278 & 196 & 10 \\
\hline Geriatrie, Gerontologie, Aging & 745 & 595 & 23 \\
\hline Epidemiologie, Public Health & 1663 & 1107 & 44 \\
\hline Summe & 6332 & 4176 & 285 \\
\hline Anteil in \% & $100 \%$ & $66,0 \%$ & $4,5 \%$ \\
\hline
\end{tabular}

Die Internetadresse wurde durch Eingabe des jeweiligen Zeitschrifttitels als Suchbegriff in Google ermittelt. Bei ca. 15\% der Titel konnte eine Internetadresse für den Volltextzugriff angegeben werden. Die Informationen bezüglich Verlag, Herausgeber und Organ wurden generell den Websites der jeweiligen Zeitschriften entnommen z.T. stark gekürzt.
Ergebnisse

\section{a) Gesamte Nennungen PMR-relevanter und fachverwandter Titel}

In Tab. 1 ist die jeweilige Anzahl der gefundenen Titel im Rahmen der Recherche angegeben, die faktisch oder potenziell PMR-relevant sind (Gesamtliste). 1871 von 6332 Titeln sind weder englisch- noch deutschsprachig (29,5\%). Einige Titel sind in mehreren Themenbereichen präsent und wurden entsprechend mehrfach gezählt.

\section{b) Auswahlliste PMR-relevanter und fachverwandter Titel}

Im Rahmen dieses Beitrags wurde auf der Basis der o.g. Recherche (Gesamtliste) in Tab. 2 eine Auswahlliste von Titeln der jeweiligen Themengebiete aufgeführt. Voraussetzung für die Aufnahme in Tab. $\mathbf{2}$ waren englisch- oder deutschsprachige Artikel im Volltext sowie das Vorhandensein einer Internetseite. Weitere Auswahlkriterien waren: Impact Factor sofern vorhanden, wissenschaftliche Relevanz und Nähe zum Fachgebiet PMR, je nach Titel und Inhalt. Die Angaben bezüglich Verlag, Herausgeber und Organ wurden möglichst kurz gefasst. Bei mehreren Internetadressen wurde die kürzeste bevorzugt, mit Ausnahme derjenigen, welche einen Volltextzugriff erlauben (jeweils vermerkt).

Weit gehend vollständig wurden in Tab. $\mathbf{2}$ die derzeit wichtigsten, primär PMR-relevanten Titel gelistet, während verwandte Fachgebiete stärker selektiert werden mussten und daher zwangsläufig auch stärker der subjektiven Wertung durch die

Tab. 2 Übersicht über Zeitschriften im Bereich der Physikalische Medizin, Rehabilitation und verwandte Fachgebiete, skaliert nach Impact Factor 2002 (IF) sowie Auswahl ohne IF (alphabetisch)

\begin{tabular}{|c|c|c|c|c|c|c|}
\hline $\begin{array}{l}\text { Themenschwerpunkt; } \\
\text { Full Title }\end{array}$ & Kurztitel/Short Title & IF 2002 & Verlag & Herausgeber/Editor & Organ/Society & Website \\
\hline \multicolumn{7}{|c|}{ Physikalische Medizin, Physikalische Therapie, Rehabilitation } \\
\hline Physical Therapy & PHYS THER & 1,658 & $\begin{array}{l}\text { Am Phys Therapy } \\
\text { Assoc }\end{array}$ & $\begin{array}{l}\text { JM Rothstein, Univ } \\
\text { of Illinois, Chicago }\end{array}$ & $\begin{array}{l}\text { Am Phys Therapy } \\
\text { Assoc }\end{array}$ & www.ptjournal.org/(Volltext) \\
\hline $\begin{array}{l}\text { Archives of Physical } \\
\text { Medicine and Rehabili- } \\
\text { tation }\end{array}$ & $\begin{array}{l}\text { ARCH PHYS MED } \\
\text { REHAB }\end{array}$ & 1,327 & $\begin{array}{l}\text { WB Saunders/Har- } \\
\text { court St. Louis MO }\end{array}$ & KM Jaffe, MD & $\begin{array}{l}\text { Am Congr of Rehab } \\
\text { Med \& Am Acad of } \\
\text { PMR }\end{array}$ & www.archives-pmr.org \\
\hline $\begin{array}{l}\text { Neuropsychological } \\
\text { Rehabilitation }\end{array}$ & $\begin{array}{l}\text { NEUROPSYCHOL } \\
\text { REHABIL }\end{array}$ & 1,200 & $\begin{array}{l}\text { Taylor \& Francis- } \\
\text { Psychology Press }\end{array}$ & $\begin{array}{l}\text { BA Wilson, Cam- } \\
\text { bridge }\end{array}$ & & $\begin{array}{l}\text { www.tandf.co.uk/journals/pp/ } \\
\text { 09602011.html }\end{array}$ \\
\hline $\begin{array}{l}\text { (Scandinavian) Journal } \\
\text { of Rehabilitation }\end{array}$ & J REHAB MED & 1,000 & Taylor \& Francis & $\begin{array}{l}\text { G Grimby, Göte- } \\
\text { borg }\end{array}$ & $\begin{array}{l}\text { Swedish Assoc of } \\
\text { Rehab and Phys } \\
\text { Med }\end{array}$ & $\begin{array}{l}\text { www.tandf.co.uk/journals/ } \\
\text { titles/16501977.asp }\end{array}$ \\
\hline $\begin{array}{l}\text { Disability and Rehabili- } \\
\text { tation }\end{array}$ & DISABIL REHABIL & 0,889 & Taylor \& Francis & $\begin{array}{l}\text { D Müller, Suffolk } \\
\text { College Ipswich }\end{array}$ & $\begin{array}{l}\text { Int Soc of Phys and } \\
\text { Rehab Med }\end{array}$ & $\begin{array}{l}\text { www.tandf.co.uk/journals/tf/ } \\
\text { 09638288.html }\end{array}$ \\
\hline Clinical Rehabilitation & CLIN REHABIL & 0,881 & Arnold Publishers & DT Wade, Oxford & $\begin{array}{l}\text { Soc for Res in } \\
\text { Rehab }\end{array}$ & $\begin{array}{l}\text { www.arnoldpublishers.com/ } \\
\text { journals/pages/cli_reh/ } \\
\text { 02692155.htm }\end{array}$ \\
\hline $\begin{array}{l}\text { American Journal of } \\
\text { Physical Medicine and } \\
\text { Rehabilitation }\end{array}$ & $\begin{array}{l}\text { AM J PHYS MED } \\
\text { REHAB }\end{array}$ & 0,877 & $\begin{array}{l}\text { Williams \& Wilkins } \\
\text { Baltimore }\end{array}$ & $\begin{array}{l}\text { B Johns, Indiana- } \\
\text { polis }\end{array}$ & $\begin{array}{l}\text { Assoc of Acad Phy- } \\
\text { siatrists }\end{array}$ & $\begin{array}{l}\text { www.amjphysmedrehab. } \\
\text { com/ }\end{array}$ \\
\hline $\begin{array}{l}\text { Rehabilitation Research } \\
\text { and Development Pro- } \\
\text { gress Reports }\end{array}$ & J REHABIL RES DEV & 0,866 & $\begin{array}{l}\text { Dept of Veterans } \\
\text { Affairs }\end{array}$ & $\begin{array}{l}\text { Dept of Veterans } \\
\text { Affairs }\end{array}$ & $\begin{array}{l}\text { Dept of Veterans } \\
\text { Affairs }\end{array}$ & $\begin{array}{l}\text { www.vard.org/prog/progindx. } \\
\text { htm }\end{array}$ \\
\hline $\begin{array}{l}\text { Journal of Orthopaedic } \\
\text { and Sports Physical } \\
\text { Therapy }\end{array}$ & $\begin{array}{l}\text { J ORTHOP SPORTS } \\
\text { PHYS THER }\end{array}$ & 0,690 & Allen Press & $\begin{array}{l}\text { GG Simoneau PT, } \\
\text { Univ Milwaukee }\end{array}$ & Am Phys Th Assoc & www.jospt.org/ \\
\hline $\begin{array}{l}\text { Journal of Occupational } \\
\text { Rehabilitation }\end{array}$ & J OCCUP REHABIL & 0,689 & Kluwer & $\begin{array}{l}\text { M Feuerstein, } \\
\text { Bethesda MD }\end{array}$ & & $\begin{array}{l}\text { www.wkap.nl/journalhome. } \\
\text { htm/1053-0487 }\end{array}$ \\
\hline Neurorehabilitation & $\begin{array}{l}\text { NEUROREHABILI- } \\
\text { TATION }\end{array}$ & 0,656 & IOS Press & $\begin{array}{l}\text { ND Zasler, Virginia } \\
\text { Univ, Richmond VA }\end{array}$ & & $\begin{array}{l}\text { www.iospress.nl/html/ } \\
\text { 10538135.html }\end{array}$ \\
\hline
\end{tabular}




\begin{tabular}{|c|c|c|c|c|c|c|}
\hline $\begin{array}{l}\text { Themenschwerpunkt; } \\
\text { Full Title }\end{array}$ & Kurztitel/Short Title & IF 2002 & Verlag & Herausgeber/Editor & Organ/Society & Website \\
\hline $\begin{array}{l}\text { Journal of Rehabilita- } \\
\text { tion }\end{array}$ & J REHABIL & 0,607 & Nat Rehab Assoc & $\begin{array}{l}\text { PP Alston, Green- } \\
\text { ville, NC }\end{array}$ & Nat Rehab Assoc & $\begin{array}{l}\text { www.nationalrehab.org/ } \\
\text { website/pubs/index.html }\end{array}$ \\
\hline $\begin{array}{l}\text { Neurorehabilitation } \\
\text { and Neural Repair }\end{array}$ & $\begin{array}{l}\text { NEUROREHAB } \\
\text { NEURAL RE }\end{array}$ & 0,592 & Demos Med Publ & ME Selzer MD PhD & $\begin{array}{l}\text { American Society } \\
\text { of Neurorehab }\end{array}$ & $\begin{array}{l}\text { www.sagepub.com/journal. } \\
\text { aspx?pid=336 }\end{array}$ \\
\hline $\begin{array}{l}\text { Physikalische Medizin, } \\
\text { Rehabilitationsmedizin, } \\
\text { Kurortmedizin }\end{array}$ & $\begin{array}{l}\text { PHYS MED REHAB } \\
\text { KUROR }\end{array}$ & 0,441 & $\begin{array}{l}\text { Thieme Verlag } \\
\text { Stuttgart }\end{array}$ & U Smolenski, Jena & $\begin{array}{l}\text { D Ges für Physikal } \\
\text { Med und Rehab }\end{array}$ & $\begin{array}{l}\text { www.thieme.de/physmed/ } \\
\text { index.html }\end{array}$ \\
\hline $\begin{array}{l}\text { International Journal of } \\
\text { Rehabilitation Research }\end{array}$ & INT J REHABIL RES & 0,366 & $\begin{array}{l}\text { Lippincott Williams } \\
\text { \& Wilkins }\end{array}$ & $\begin{array}{l}\text { J Wikström, } \\
\text { Finland }\end{array}$ & $\begin{array}{l}\text { Eur Fed for Res in } \\
\text { Rehab }\end{array}$ & www.intjrehabilres.com/ \\
\hline $\begin{array}{l}\text { Journal of Back and } \\
\text { Musculo-skeletal Reha- } \\
\text { bilitation }\end{array}$ & $\begin{array}{l}\text { J BACK MUSCU- } \\
\text { LOSKELET }\end{array}$ & 0,089 & $\begin{array}{l}\text { IOS Press Amster- } \\
\text { dam }\end{array}$ & $\begin{array}{l}\text { H. Hermens, } \\
\text { Enschede (NL) }\end{array}$ & & $\begin{array}{l}\text { www.iospress.nl/html/ } \\
\text { 10538127.html }\end{array}$ \\
\hline & & kein IF & & & & \\
\hline $\begin{array}{l}\text { Critical Reviews in } \\
\text { Physical \& Rehabilita- } \\
\text { tion Medicine }\end{array}$ & $\begin{array}{l}\text { Crit Rev Phys } \\
\text { Rehab Med }\end{array}$ & 0 & $\begin{array}{l}\text { Begell House Inc, } \\
\text { New York }\end{array}$ & $\begin{array}{l}\text { M Grabois, EJ Hen- } \\
\text { ley, Univ Houston }\end{array}$ & & $\begin{array}{l}\text { www.begellhouse.com/ } \\
\text { crprm/crprm.html }\end{array}$ \\
\hline Die Rehabilitation & $\begin{array}{l}\text { Rehabilitation } \\
\text { (Stuttg) }\end{array}$ & 0 & Thieme Stuttgart & $\begin{array}{l}\text { KA Jochheim, Erft- } \\
\text { stadt-Lechenich }\end{array}$ & $\begin{array}{l}\text { D Vereinigung für } \\
\text { Rehab Behinderter }\end{array}$ & $\begin{array}{l}\text { www.thieme.de/rehabilitation } \\
\text { /index.html }\end{array}$ \\
\hline $\begin{array}{l}\text { International Journal } \\
\text { of Rehabilitation and } \\
\text { Health }\end{array}$ & Int J Rehab Health & 0 & Kluwer & $\begin{array}{l}\text { AJ Goreczny, Pitts- } \\
\text { burgh, USA }\end{array}$ & & $\begin{array}{l}\text { www.wkap.nl/journalhome. } \\
\text { htm/1068-9591 }\end{array}$ \\
\hline $\begin{array}{l}\text { International Journal of } \\
\text { Rehabilitation Review }\end{array}$ & Int J Rehabil Rev & 0 & Rehab Int & B Duncan & & $\begin{array}{l}\text { www.rehab-international.org/ } \\
\text { toc.html }\end{array}$ \\
\hline International Journal of & $\mathrm{Br}(\ln \mathrm{t}) \mathrm{J}$ Ther & 0 & Mark Allen Publ & A Rushton, MSc. & & www.ijtr.co.uk/ \\
\hline $\begin{array}{l}\text { Therapy and Rehabilita- } \\
\text { tion }\end{array}$ & Rehabil & & & & & \\
\hline $\begin{array}{l}\text { Journal of Cardiopul- } \\
\text { monary Rehabilitation } \\
\text { (USA) }\end{array}$ & J Cardpulm Rehabil & 0 & $\begin{array}{l}\text { Lippincott Williams } \\
\& \text { Wilkins }\end{array}$ & $\begin{array}{l}\text { G] Balady, MD/PA } \\
\text { Ades, MD }\end{array}$ & $\begin{array}{l}\text { Am Assoc of Car- } \\
\text { diovasc and Pul- } \\
\text { mon Rehab }\end{array}$ & $\begin{array}{l}\text { www.lww.com/productdetail } \\
\text { results/1,2265,33811462,00. } \\
\text { html }\end{array}$ \\
\hline $\begin{array}{l}\text { Journal of Sport Chiro- } \\
\text { practic and Rehabilita- } \\
\text { tion (USA) }\end{array}$ & $\begin{array}{l}\text { J Sport Chiropr } \\
\text { Rehab }\end{array}$ & 0 & $\begin{array}{l}\text { Lippincott Williams } \\
\& \text { Wilkins }\end{array}$ & & & www.acasc.org/JSC.html \\
\hline $\begin{array}{l}\text { Journal of Sport Reha- } \\
\text { bilitation (USA) }\end{array}$ & J Sport Rehab & 0 & Human Kinetics & $\begin{array}{l}\text { CD Ingersoll, PhD, } \\
\text { Univ of Virginia }\end{array}$ & & $\begin{array}{l}\text { www.humankinetics.com/ } \\
\text { products/journals/journal. } \\
\text { cfm?id=JSR }\end{array}$ \\
\hline $\begin{array}{l}\text { Orthopaedic Physical } \\
\text { Therapy Clinics of } \\
\text { North America }\end{array}$ & $\begin{array}{l}\text { Orthop Phys Ther } \\
\text { Clin N Am }\end{array}$ & 0 & $\begin{array}{l}\text { WB Saunders/Har- } \\
\text { court St. Louis MO }\end{array}$ & & & $\begin{array}{l}\text { www.harcourthealth.com/ } \\
\text { scripts/om.dll/serve?action= } \\
\text { searchDB\&searchDBfor=home } \\
\text { \&id=copt }\end{array}$ \\
\hline $\begin{array}{l}\text { Oester Zeitschrift für } \\
\text { Physikal. Medizin und } \\
\text { Rehabilitation }\end{array}$ & $\begin{array}{l}\text { ÖZPMR, Österr Z } \\
\text { Phys Med Rehabil }\end{array}$ & 0 & Uhlen Verlag & K Ammer, Wien & $\begin{array}{l}\text { BÖPMR_-Berufs- } \\
\text { verb Österr. Fach- } \\
\text { ärzte für PMR }\end{array}$ & $\begin{array}{l}\text { www.boepmr.at/html/aerzte/ } \\
\text { zeitung/1zeitung/inhalt_ } \\
1 \text { 01.htm }\end{array}$ \\
\hline $\begin{array}{l}\text { Physical Medicine and } \\
\text { Rehabil. Clinics of } \mathrm{N} \\
\text { America }\end{array}$ & $\begin{array}{l}\text { Phys Med Rehab } \\
\text { Clin }\end{array}$ & 0 & $\begin{array}{l}\text { WB Saunders/Har- } \\
\text { court Health Sci- } \\
\text { ences }\end{array}$ & & & http://pmr.theclinics.com/ \\
\hline $\begin{array}{l}\text { Physical Therapy Case } \\
\text { Reports }\end{array}$ & Phys Ther Case Rep & 0 & $\begin{array}{l}\text { LRP/Lippincott Wil- } \\
\text { liams \& Wilkins }\end{array}$ & & & $\begin{array}{l}\text { http://lww.com/product } \\
\text { detailresults/1,2265,1094- } \\
\text { 0367,00.html }\end{array}$ \\
\hline $\begin{array}{l}\text { Physical Therapy in } \\
\text { Sport (UK) }\end{array}$ & Phys Ther Sport & 0 & $\begin{array}{l}\text { Churchill Living- } \\
\text { stone/Harcourt In- } \\
\text { ternational }\end{array}$ & $\begin{array}{l}\text { K Foreman, Univ of } \\
\text { the West Bristol, } \\
\text { UK }\end{array}$ & $\begin{array}{l}\text { Assoc of Chartered } \\
\text { Physiotherapists in } \\
\text { Sports Med }\end{array}$ & $\begin{array}{l}\text { www.harcourt-international. } \\
\text { com/journals/ptsp/ }\end{array}$ \\
\hline $\begin{array}{l}\text { Physical Therapy } \\
\text { Reviews }\end{array}$ & Phys Ther Rev & 0 & $\begin{array}{l}\text { WB Saunders/Ma- } \\
\text { ney }\end{array}$ & $\begin{array}{l}\text { GD Baxter, Univ of } \\
\text { Ulster, UK }\end{array}$ & & $\begin{array}{l}\text { www.maney.co.uk/search? } \\
\text { fwaction=show\&fwid=197 }\end{array}$ \\
\hline $\begin{array}{l}\text { Springer Series in } \\
\text { Rehabilitation }\end{array}$ & $\begin{array}{l}\text { Springer Ser Reha- } \\
\text { bil }\end{array}$ & 0 & $\begin{array}{l}\text { Springer Berlin } \\
\text { Heidelberg }\end{array}$ & MG Eisenberg PhD & & $\begin{array}{l}\text { www.springerpub.com/store/ } \\
\text { SSR.html }\end{array}$ \\
\hline $\begin{array}{l}\text { Topics in SCI (Spinal } \\
\text { Cord Injury) Rehabili- } \\
\text { tation }\end{array}$ & $\begin{array}{l}\text { Top Spinal Cord Inj } \\
\text { Rehabil }\end{array}$ & 0 & $\begin{array}{l}\text { Thomas Land Publ, } \\
\text { Inc. }\end{array}$ & $\begin{array}{l}\text { DF Apple Jr MD, } \\
\text { Atlanta, Georgia }\end{array}$ & & www.thomasland.com/ \\
\hline $\begin{array}{l}\text { Topics in Stroke Reha- } \\
\text { bilitation }\end{array}$ & Top Stroke Rehabil & 0 & $\begin{array}{l}\text { Thomas Land Publ, } \\
\text { Inc. }\end{array}$ & $\begin{array}{l}\text { EJ Roth MD, Dir Re- } \\
\text { hab Inst of Chicago }\end{array}$ & & www.thomasland.com/ \\
\hline \multicolumn{7}{|c|}{ Physiotherapie („Krankengymnastik“), Ergotherapie (= Occupational Therapy) } \\
\hline Physical Therapy & PHYS THER & 1,658 & $\begin{array}{l}\text { Am Phys Therapy } \\
\text { Assoc }\end{array}$ & $\begin{array}{l}\text { JM Rothstein, Univ } \\
\text { of Illinois, Chicago }\end{array}$ & $\begin{array}{l}\text { Am Phys Therapy } \\
\text { Assoc }\end{array}$ & www.ptjournal.org/(Volltext) \\
\hline $\begin{array}{l}\text { Journal of Orthopaedic } \\
\text { and Sports Physical } \\
\text { Therapy }\end{array}$ & $\begin{array}{l}\text { J ORTHOP SPORTS } \\
\text { PHYS THER }\end{array}$ & 0,690 & Allen Press & $\begin{array}{l}\text { GG Simoneau PT, } \\
\text { Univ Milwaukee }\end{array}$ & Am Phys Th Assoc & www.jospt.org/ \\
\hline $\begin{array}{l}\text { American Journal of } \\
\text { Occupational Therapy }\end{array}$ & AM J OCCUP THER & 0,550 & $\begin{array}{l}\text { Am Occup Ther } \\
\text { Assoc }\end{array}$ & & $\begin{array}{l}\text { Am Occup Ther } \\
\text { Assoc }\end{array}$ & $\begin{array}{l}\text { www.aota.org/nonmembers/ } \\
\text { area7/links/LINK03.asp }\end{array}$ \\
\hline $\begin{array}{l}\text { Australian Journal of } \\
\text { Physiotherapy }\end{array}$ & AUST J PHYSIOTH & 0,375 & $\begin{array}{l}\text { Austr Physioth As- } \\
\text { soc }\end{array}$ & M Galea PhD & $\begin{array}{l}\text { Austr Physioth As- } \\
\text { soc }\end{array}$ & $\begin{array}{l}\text { www.physiotherapy.asn.au/ } \\
\text { AJP/(Volltext) }\end{array}$ \\
\hline
\end{tabular}




Themenschwerpunkt; Kurztitel/Short Title IF $2002 \quad$ Verlag Website Organ/Society
Full Title

\begin{tabular}{|c|c|c|c|c|c|c|}
\hline & & kein IF & & & & \\
\hline $\begin{array}{l}\text { Advances in Physio- } \\
\text { therapy }\end{array}$ & Adv Physiother & 0 & Taylor \& Francis & $\begin{array}{l}\text { M Abrandt Dahl- } \\
\text { gren, Sweden }\end{array}$ & & $\begin{array}{l}\text { www.tandf.co.uk/journals/tfs/ } \\
\text { 14038196.html }\end{array}$ \\
\hline $\begin{array}{l}\text { Australian Occupation- } \\
\text { al Therapy Journal }\end{array}$ & Austr Occup Ther J & 0 & Blackwell Science & $\begin{array}{l}\text { J Fricke, La Trobe } \\
\text { Univ, Melbourne }\end{array}$ & $\begin{array}{l}\text { Austr Assoc of Oc- } \\
\text { cup Therapists. }\end{array}$ & $\begin{array}{l}\text { www.blackwellpublishing. } \\
\text { com/jour- } \\
\text { nal.asp?ref=0045-0766\& } \\
\text { site=1 }\end{array}$ \\
\hline $\begin{array}{l}\text { Ergotherapie (Öster- } \\
\text { reich) }\end{array}$ & & 0 & & C Wenger & $\begin{array}{l}\text { Verb der Ergothe- } \\
\text { rapeut. Österreichs }\end{array}$ & $\begin{array}{l}\text { www.ergotherapie.at/ } \\
\text { I_Ztg.htm }\end{array}$ \\
\hline Ergotherapie (Schweiz) & & 0 & Stämpfli AG, Bern & C Galli, Zürich & $\begin{array}{l}\text { Ergotherapeutln- } \\
\text { nen-Verb Schweiz }\end{array}$ & $\begin{array}{l}\text { www.ergotherapie.ch/ } \\
\text { zeit_impressum.html }\end{array}$ \\
\hline $\begin{array}{l}\text { Ergotherapie \& Reha- } \\
\text { bilitation }\end{array}$ & & 0 & $\begin{array}{l}\text { D Verb der Ergo- } \\
\text { ther e. V. }\end{array}$ & $\begin{array}{l}\text { C Berting-Hüneke, } \\
\text { Hannover }\end{array}$ & $\begin{array}{l}\text { D Verb der Ergo- } \\
\text { ther eV }\end{array}$ & $\begin{array}{l}\text { www.schulz-kirchner.de/ } \\
\text { ergotherapie/zeitschrift.htm }\end{array}$ \\
\hline $\begin{array}{l}\text { Journal für Ergothera- } \\
\text { peutische Forschung } \\
\text { und Lehre }\end{array}$ & & 0 & $\begin{array}{l}\text { Dr. Udo Häusler, } \\
\text { Göttingen }\end{array}$ & $\begin{array}{l}\text { Dr. U Häusler, Göt- } \\
\text { tingen }\end{array}$ & & $\begin{array}{l}\text { www.ergotherapy.de/index. } \\
\text { htm }\end{array}$ \\
\hline $\begin{array}{l}\text { Journal of Bodywork } \\
\text { and Movement Thera- } \\
\text { pies }\end{array}$ & $\begin{array}{l}\text { J Bodywork Mov } \\
\text { Ther }\end{array}$ & 0 & $\begin{array}{l}\text { Harcourt Inter- } \\
\text { national }\end{array}$ & L Chaitow & $\begin{array}{l}\text { Irish Ass of Phys } \\
\text { Therapists }\end{array}$ & $\begin{array}{l}\text { www.harcourt-international. } \\
\text { com/journals/jbmt/ }\end{array}$ \\
\hline $\begin{array}{l}\text { Krankengymnastik - } \\
\text { Zeitschrift für Physio- } \\
\text { therapeuten }\end{array}$ & Krankengymnastik & 0 & $\begin{array}{l}\text { Pflaum Verlag } \\
\text { GmbH, München }\end{array}$ & A Hüter-Becker & & $\begin{array}{l}\text { www.pflaum.de/pt.dir/kg/ } \\
\text { index.html }\end{array}$ \\
\hline $\begin{array}{l}\text { Orthopaedic Physical } \\
\text { Therapy Clinics of } \\
\text { North America }\end{array}$ & $\begin{array}{l}\text { Orthop Phys Ther } \\
\text { Clin N Am }\end{array}$ & 0 & $\begin{array}{l}\text { WB Saunders/Har- } \\
\text { court St. Louis MO }\end{array}$ & & & $\begin{array}{l}\text { www.harcourthealth.com/ } \\
\text { scripts/om.dll/serve?action= } \\
\text { searchDB\&searchDBfor=home } \\
\text { \&id=copt }\end{array}$ \\
\hline $\begin{array}{l}\text { Physical Therapy Case } \\
\text { Reports }\end{array}$ & Phys Ther Case Rep & 0 & $\begin{array}{l}\text { LRP/Lippincott Wil- } \\
\text { liams \& Wilkins }\end{array}$ & & & $\begin{array}{l}\text { http://lww.com/product } \\
\text { detailresults/ } \\
1,2265,1094-0367,00 . h t m \mid\end{array}$ \\
\hline $\begin{array}{l}\text { Physical Therapy in } \\
\text { Sport (UK) }\end{array}$ & Phys Ther Sport & 0 & $\begin{array}{l}\text { Churchill Living- } \\
\text { stone/Harcourt } \\
\text { International }\end{array}$ & $\begin{array}{l}\text { K Foreman, Univ of } \\
\text { the West Bristol, } \\
\text { UK }\end{array}$ & $\begin{array}{l}\text { Assoc of Chartered } \\
\text { Physiotherapists in } \\
\text { Sports Med }\end{array}$ & $\begin{array}{l}\text { www.harcourt-international. } \\
\text { com/journals/ptsp/ }\end{array}$ \\
\hline $\begin{array}{l}\text { Physical Therapy } \\
\text { Reviews }\end{array}$ & Phys Ther Rev & 0 & $\begin{array}{l}\text { WB Saunders/ } \\
\text { Maney }\end{array}$ & $\begin{array}{l}\text { GD Baxter, Univ of } \\
\text { Ulster, UK }\end{array}$ & & $\begin{array}{l}\text { www.maney.co.uk/search? } \\
\text { fwaction=show\&fwid=197 }\end{array}$ \\
\hline $\begin{array}{l}\text { Physiotherapy Research } \\
\text { International }\end{array}$ & Physiother Res Int & 0 & $\begin{array}{l}\text { Whurr Publishers } \\
\text { Ltd }\end{array}$ & $\begin{array}{l}\text { Dr C Partridge, } \\
\text { CHSS, Canterbury }\end{array}$ & & www.whurrjournals.co.uk/pri \\
\hline $\begin{array}{l}\text { Physiotherapy Theory } \\
\text { and Practice }\end{array}$ & $\begin{array}{l}\text { Physiother Theory } \\
\text { Pract }\end{array}$ & 0 & $\begin{array}{l}\text { Lawrence Erlbaum } \\
\text { Assoc }\end{array}$ & $\begin{array}{l}\text { S Hasson, Univ of } \\
\text { Connecticut }\end{array}$ & & $\begin{array}{l}\text { www.tandf.co.uk/journals/tf/ } \\
\text { 09593985.html }\end{array}$ \\
\hline \multicolumn{7}{|c|}{ Manuelle Therapie, Massage, Lymphologie } \\
\hline $\begin{array}{l}\text { Journal of Manipulative } \\
\text { and Physiological Ther- } \\
\text { apeutics }\end{array}$ & $\begin{array}{l}\text { J MANIP PHYSIOL } \\
\text { THER }\end{array}$ & 1,041 & Mosby, Inc. & DJ Lawrence, DC & & www.mosby.com/jmpt \\
\hline Manual Therapy & MANUAL THER & 0.796 & Elsevier & $\begin{array}{l}\text { Prof A Moore, Ass } \\
\text { Prof } G \text { Jull }\end{array}$ & $\begin{array}{l}\text { Manip. Assoc of } \\
\text { Chartered Physio- } \\
\text { therapists }\end{array}$ & $\begin{array}{l}\text { www.harcourt-international. } \\
\text { com/journals/math/ }\end{array}$ \\
\hline Lymphology & LYMPHOLOGY & $\begin{array}{l}0,750 \\
\text { kein IF }\end{array}$ & Lymphology & $\begin{array}{l}\text { CL Witte, Tucson, } \\
\text { Arizona, USA }\end{array}$ & $\begin{array}{l}\text { Int Soc of Lympho- } \\
\text { logy }\end{array}$ & $\begin{array}{l}\text { http://www.u.arizona.edu/ } \\
\sim \text { witte/journal.htm }\end{array}$ \\
\hline $\begin{array}{l}\text { Journal of Manual and } \\
\text { Manipulative Therapy }\end{array}$ & J Man Manip Ther & 0 & $\begin{array}{l}\text { J of Man and } \\
\text { Manip Ther }\end{array}$ & $\begin{array}{l}\text { JM Medeiros, PT } \\
\text { Ph.D }\end{array}$ & $\begin{array}{l}\text { Am Acad of Orthop } \\
\text { Man Phys Thera- } \\
\text { pists }\end{array}$ & http://home1.gte.net/jmmt/ \\
\hline Manuelle Medizin & Man Med & 0 & $\begin{array}{l}\text { Springer Heidel- } \\
\text { berg }\end{array}$ & $\begin{array}{l}\text { G Gallacchi, W Gil- } \\
\text { liar, U Smolenski }\end{array}$ & $\begin{array}{l}\text { Ärzteverein für Ma- } \\
\text { nuelle Medizin - } \\
\text { e.V. }\end{array}$ & $\begin{array}{l}\text { www.springerlink.com/ } \\
\text { openurl.asp?genre=jour- } \\
\text { nal\&issn=0025-2514 }\end{array}$ \\
\hline Manuelle Therapie & & 0 & $\begin{array}{l}\text { Thieme Verlag } \\
\text { Stuttgart }\end{array}$ & $\begin{array}{l}\text { B Falkenberg, Iser- } \\
\text { lohn, }\end{array}$ & & $\begin{array}{l}\text { www.thieme.de/manuelle- } \\
\text { therapie/index.html }\end{array}$ \\
\hline $\begin{array}{l}\text { Massage Therapy } \\
\text { Journal }\end{array}$ & & 0 & $\begin{array}{l}\text { American Massage } \\
\text { Therapy Associa- } \\
\text { tion }\end{array}$ & M Schwantz & $\begin{array}{l}\text { Am Massage Ther } \\
\text { Assoc }\end{array}$ & $\begin{array}{l}\text { www.amtamassage.org/ } \\
\text { journal/ }\end{array}$ \\
\hline \multicolumn{7}{|c|}{ Kurortmedizin, Balneotherapie, Thermotherapie, Med. Klimatologie } \\
\hline $\begin{array}{l}\text { International Journal } \\
\text { of Biometeorology }\end{array}$ & $\begin{array}{l}\text { INT J BIOMETEO- } \\
\text { ROL }\end{array}$ & 1,273 & Springer Verlag & Prof. M Iriki & $\begin{array}{l}\text { Int Soc of Biome- } \\
\text { teorol }\end{array}$ & $\begin{array}{l}\text { http://link.springer.de/link/ } \\
\text { service/journals/00484/ }\end{array}$ \\
\hline $\begin{array}{l}\text { Physikalische Medizin, } \\
\text { Rehabilitationsmedizin, } \\
\text { Kurortmedizin }\end{array}$ & $\begin{array}{l}\text { PHYS MED REHAB } \\
\text { KUROR }\end{array}$ & 0,441 & $\begin{array}{l}\text { Thieme Verlag } \\
\text { Stuttgart }\end{array}$ & U Smolenski, Jena & $\begin{array}{l}\text { D Ges für Physikal } \\
\text { Med und Rehab }\end{array}$ & $\begin{array}{l}\text { www.thieme.de/physmed/ } \\
\text { index.html }\end{array}$ \\
\hline $\begin{array}{l}\text { Thermology Internatio- } \\
\text { nal (former European } \\
\text { Journal of Thermology; } \\
\text { Austria) }\end{array}$ & Thermol Int & $\begin{array}{l}\text { kein IF } \\
0\end{array}$ & Uhlen & K Ammer, Wien & $\begin{array}{l}\text { European Associa- } \\
\text { tion of Thermology }\end{array}$ & $\begin{array}{l}\text { www.uhlen.at/thermology- } \\
\text { international/ }\end{array}$ \\
\hline
\end{tabular}




\begin{tabular}{|c|c|c|c|c|c|c|}
\hline $\begin{array}{l}\text { Themenschwerpunkt; } \\
\text { Full Title }\end{array}$ & Kurztitel/Short Title & IF 2002 & Verlag & Herausgeber/Editor & Organ/Society & Website \\
\hline \multicolumn{7}{|c|}{ Orthopädie, Rheumatologie } \\
\hline $\begin{array}{l}\text { Arthritis and Rheuma- } \\
\text { tism }\end{array}$ & ARTHRITIS RHEUM & 7,379 & John Wiley \& Sons & $\begin{array}{l}\text { DS Pisetsky, MD, } \\
\text { PhD }\end{array}$ & $\begin{array}{l}\text { Am College of } \\
\text { Rheum (ACR) }\end{array}$ & $\begin{array}{l}\text { www.rheumatology.org/ar/ } \\
\text { ar.html }\end{array}$ \\
\hline $\begin{array}{l}\text { Annals of the Rheuma- } \\
\text { tic Diseases }\end{array}$ & ANN RHEUM DIS & 3,593 & $\begin{array}{l}\text { British Med J Publ } \\
\text { Group }\end{array}$ & $\begin{array}{l}\text { Edit Ass Univ } \\
\text { Nijmegen, NL }\end{array}$ & $\begin{array}{l}\text { Eur League Against } \\
\text { Rheum (Eular) }\end{array}$ & http://ard.bmjjournals.com/ \\
\hline $\begin{array}{l}\text { Rheumatic Disease } \\
\text { Clinics of North Ameri- } \\
\text { ca }\end{array}$ & $\begin{array}{l}\text { RHEUM DIS CLIN N } \\
\text { AM }\end{array}$ & 3,314 & WB Saunders Co. & & & $\begin{array}{l}\text { http://rheumatic.theclinics. } \\
\text { com/ }\end{array}$ \\
\hline Rheumatology & RHEUMATOLOGY & 3,251 & $\begin{array}{l}\text { Oxford Univ Press } \\
\text { (OUP) }\end{array}$ & $\begin{array}{l}\text { Dr DL Scott, Lon- } \\
\text { don }\end{array}$ & $\begin{array}{l}\text { British Soc for } \\
\text { Rheum }\end{array}$ & $\begin{array}{l}\text { http://rheumatology. } \\
\text { oupjournals.org/ }\end{array}$ \\
\hline $\begin{array}{l}\text { Journal of Rheumato- } \\
\text { logy }\end{array}$ & J RHEUMATOL & 2,987 & $\begin{array}{l}\text { J Rheumatol Publ } \\
\text { Co., Toronto }\end{array}$ & DA Gordon & & www.jrheum.com/ \\
\hline $\begin{array}{l}\text { Seminars in Arthritis } \\
\text { and Rheumatism }\end{array}$ & SEM ARTH RHEU & 2,750 & WB Saunders Co. & $\begin{array}{l}\text { RD Altman, MD, NL } \\
\text { Gottlieb, MD }\end{array}$ & & $\begin{array}{l}\text { www.sciencedirect.com/ } \\
\text { science/journal/00490172 } \\
\text { (Volltext) }\end{array}$ \\
\hline $\begin{array}{l}\text { Osteoporosis Interna- } \\
\text { tional }\end{array}$ & $\begin{array}{l}\text { OSTEOPOROSIS } \\
\text { INT }\end{array}$ & $\begin{array}{l}2,613 \\
(2000)\end{array}$ & Springer Verlag & $\begin{array}{l}\text { R Lindsay, PJ Meu- } \\
\text { nier }\end{array}$ & $\begin{array}{l}\text { Int Osteoporosis } \\
\text { Found of the USA }\end{array}$ & $\begin{array}{l}\text { http://link.springer.de/link/ } \\
\text { service/journals/00198/index. } \\
\text { htm }\end{array}$ \\
\hline Spine & SPINE & 2,311 & $\begin{array}{l}\text { Lippincott Williams } \\
\text { \& Wilkins }\end{array}$ & $\begin{array}{l}\text { JN Weinstein, DO, } \\
\text { MS }\end{array}$ & $\begin{array}{l}\text { Cervical Spine Res } \\
\text { Soc }\end{array}$ & www.spinejournal.com \\
\hline $\begin{array}{l}\text { Journal of Bone and } \\
\text { Joint Surgery (Am Vol) }\end{array}$ & $\begin{array}{l}\text { J BONE JOINT SURG } \\
\text { AM }\end{array}$ & 2,046 & $\begin{array}{l}\text { J of Bone and Joint } \\
\text { Surg Inc }\end{array}$ & JD Heckman & $\begin{array}{l}\text { The Am Orthop } \\
\text { Assoc }\end{array}$ & www.ejbjs.org \\
\hline $\begin{array}{l}\text { Scandinavian Journal } \\
\text { of Rheumatology }\end{array}$ & $\begin{array}{l}\text { SCAND J RHEUMA- } \\
\text { TOL }\end{array}$ & 2,000 & Taylor \& Francis AS & G Husby, Oslo & & $\begin{array}{l}\text { www.tandf.co.uk/journals/tfs/ } \\
\text { 03009742.html }\end{array}$ \\
\hline $\begin{array}{l}\text { Journal of Orthopaedic } \\
\text { Research }\end{array}$ & J ORTHOPAED RES & 1,846 & Elsevier Science & $\begin{array}{l}\text { JA Buckwater, MD, } \\
\text { Univ of lowa }\end{array}$ & & $\begin{array}{l}\text { www.sciencedirect.com/ } \\
\text { science/journal/07360266 } \\
\text { (Volltext) }\end{array}$ \\
\hline $\begin{array}{l}\text { Arthritis Care and } \\
\text { Research }\end{array}$ & ARTHRIT CARE RES & 1,811 & $\begin{array}{l}\text { Lippincott Williams } \\
\& \text { Wilkins }\end{array}$ & $\begin{array}{l}\text { GG Hunder, MD } \\
\text { Rochester, MN }\end{array}$ & $\begin{array}{l}\text { Am College of } \\
\text { Rheum }\end{array}$ & $\begin{array}{l}\text { www.interscience.wiley.com/ } \\
\text { jpages/0004-3591+/ }\end{array}$ \\
\hline $\begin{array}{l}\text { The Journal of Bone and } \\
\text { Joint Surgery ( } \mathrm{Br} \text { Vol) }\end{array}$ & $\begin{array}{l}\text { J BONE JOINT SURG } \\
\text { BR }\end{array}$ & 1,457 & $\begin{array}{l}\text { Br Edit Soc of Bone } \\
\text { and Joint Surg }\end{array}$ & F Horan & $\begin{array}{l}\text { Br Edit Soc of Bone } \\
\text { and Joint Surg }\end{array}$ & www.jbjs.org.uk \\
\hline $\begin{array}{l}\text { Clinical and Experimen- } \\
\text { tal Rheumatology }\end{array}$ & $\begin{array}{l}\text { CLIN EXP RHEUMA- } \\
\text { TOL }\end{array}$ & 1,284 & Clin \& Exp Rheum & S Bombardieri, Pisa & $\begin{array}{l}\text { „editorial inde- } \\
\text { pendence“ }\end{array}$ & www.clinexprheumatol.org/ \\
\hline $\begin{array}{l}\text { Clinical Orthopaedics } \\
\text { and Related Research }\end{array}$ & $\begin{array}{l}\text { CLIN ORTHOP RE- } \\
\text { LAT R }\end{array}$ & 1,208 & $\begin{array}{l}\text { Lippincott Williams } \\
\text { \& Wilkins }\end{array}$ & $\begin{array}{l}\text { CT Brighton, Phila- } \\
\text { delphia }\end{array}$ & $\begin{array}{l}\text { Assoc of Bone and } \\
\text { Joint Surg }\end{array}$ & www.corronline.com \\
\hline Acta Orthopaedica & ACTA ORTHOP & 1,169 & Taylor \& Francis & A Rydholm, Lund & Nordic Orthop Fed & www.catchword.com/rpsv/ \\
\hline Scandinavica & SCAND & & & & & $\begin{array}{l}\text { cw/tandf/00016470/ } \\
\text { contp1.htm (Volltext) }\end{array}$ \\
\hline $\begin{array}{l}\text { Rheumatology Interna- } \\
\text { tional }\end{array}$ & RHEUMATOL INT & 1,000 & Springer Verlag & $\begin{array}{l}\text { Prof E-M Lemmel, } \\
\text { Brühl }\end{array}$ & & $\begin{array}{l}\text { http://link.springer.de/link/ } \\
\text { service/journals/00296/index. } \\
\text { htm }\end{array}$ \\
\hline Clinical Rheumatology & CLIN RHEUMATOL & 0,976 & $\begin{array}{l}\text { Springer Verlag } \\
\text { (ASSMB) }\end{array}$ & J Dequeker & Belgian Rheum Soc & $\begin{array}{l}\text { http://link.springer.de/link/ } \\
\text { service/journals/10067/index. } \\
\text { htm }\end{array}$ \\
\hline $\begin{array}{l}\text { Zeitschrift für Rheuma- } \\
\text { tologie }\end{array}$ & Z RHEUMATOL & 0,964 & $\begin{array}{l}\text { Dr. Dietrich Stein- } \\
\text { kopff Verlag Darm- } \\
\text { stadt }\end{array}$ & $\begin{array}{l}\text { Prof Dr WL Gross, } \\
\text { Uniklinik Lübeck }\end{array}$ & $\begin{array}{l}\text { D, Oesterr + } \\
\text { Schweizer Ges. f. } \\
\text { Rheum }\end{array}$ & $\begin{array}{l}\text { http://link.springer-ny.com/ } \\
\text { link/service/journals/00393/ }\end{array}$ \\
\hline $\begin{array}{l}\text { Journal of Orthopaedic } \\
\text { Trauma }\end{array}$ & J ORTHOP TRAUMA & 0,929 & $\begin{array}{l}\text { Lippincott Williams } \\
\text { \& Wilkins }\end{array}$ & $\begin{array}{l}\text { RW Sanders, Tam- } \\
\text { pa }\end{array}$ & $\begin{array}{l}\text { Orthop Trauma } \\
\text { Assoc }\end{array}$ & www.jorthotrauma.com/ \\
\hline Joint Bone Spine & JOINT BONE SPINE & 0,675 & $\begin{array}{l}\text { Editions Scientifi- } \\
\text { ques Med Elsevier }\end{array}$ & M-C Boissier, Paris & $\begin{array}{l}\text { French Soc of } \\
\text { Rheum }\end{array}$ & $\begin{array}{l}\text { www.jointbonespine.com/ } \\
\text { (Volltext) }\end{array}$ \\
\hline $\begin{array}{l}\text { Baillieres Best Practice } \\
\text { \& Research in Clin. } \\
\text { Rheumatology }\end{array}$ & $\begin{array}{l}\text { BEST PRACT RES CL } \\
\text { RH }\end{array}$ & 0,646 & $\begin{array}{l}\text { Bailliere Tindall/ } \\
\text { Harcourt Health Sc }\end{array}$ & $\begin{array}{l}\text { AD Woolf, Royal } \\
\text { Hospital, Truro }\end{array}$ & & $\begin{array}{l}\text { www.harcourt-international. } \\
\text { com/journals/berh/ }\end{array}$ \\
\hline Orthopäde (Der) & ORTHOPADE & 0,583 & Springer Verlag & $\begin{array}{l}\text { V Ewerbeck, Hei- } \\
\text { delberg }\end{array}$ & & $\begin{array}{l}\text { http://link.springer.de/link/ } \\
\text { service/journals/00132/ }\end{array}$ \\
\hline $\begin{array}{l}\text { Zeitschrift für Ortho- } \\
\text { pädie und ihre Grenz- } \\
\text { gebiete }\end{array}$ & $\begin{array}{l}\text { Z ORTHOP GRENZ- } \\
\text { GEB }\end{array}$ & 0,529 & Thieme Verlag & $\begin{array}{l}\text { MH Hackenbroch, } \\
\text { Köln/R Kotz, Wien }\end{array}$ & $\begin{array}{l}\text { D Gesellschaft für } \\
\text { Orthop und Traum }\end{array}$ & www.thieme.de/zfo/ \\
\hline $\begin{array}{l}\text { JCR - Journal of Clinical } \\
\text { Rheumatology }\end{array}$ & $\begin{array}{l}\text { JCR-J CLIN RHEU- } \\
\text { MATOL }\end{array}$ & 0,455 & $\begin{array}{l}\text { Lippincott Williams } \\
\& \text { Wilkins }\end{array}$ & $\begin{array}{l}\text { HR Schumacher Jr, } \\
\text { MD }\end{array}$ & PANLAR & www.jclinrheum.com/ \\
\hline $\begin{array}{l}\text { Prosthetics and Ortho- } \\
\text { tics International }\end{array}$ & $\begin{array}{l}\text { PROSTHET OR- } \\
\text { THOT INT }\end{array}$ & 0,393 & $\begin{array}{l}\text { Int Soc of Prosthet } \\
\text { and Orthot }\end{array}$ & J Hughes, N Jacobs & $\begin{array}{l}\text { Int Soc of Prosthet } \\
\text { and Orthot }\end{array}$ & $\begin{array}{l}\text { www.ispo.ws/HTML/Page- } \\
\text { Journal.html }\end{array}$ \\
\hline $\begin{array}{l}\text { Aktuelle Rheumato- } \\
\text { logie }\end{array}$ & $\begin{array}{l}\text { AKTUEL RHEUMA- } \\
\text { TOL }\end{array}$ & 0,370 & Thieme Verlag & $\begin{array}{l}\text { W Miehle, Bad Aib- } \\
\text { ling }\end{array}$ & & $\begin{array}{l}\text { www.thieme.de/rheuma/ } \\
\text { index.html }\end{array}$ \\
\hline Orthopedics & ORTHOPEDICS & 0,267 & Slack Inc & $\begin{array}{l}\text { R D’Ambrosia, MD, } \\
\text { New Orleans }\end{array}$ & & www.orthobluejournal.com \\
\hline
\end{tabular}




\begin{tabular}{|c|c|c|c|c|c|c|}
\hline $\begin{array}{l}\text { Themenschwerpunkt; } \\
\text { Full Title }\end{array}$ & Kurztitel/Short Title & IF 2002 & Verlag & Herausgeber/Editor & Organ/Society & Website \\
\hline \multicolumn{7}{|l|}{ Neurologie, Schmerz } \\
\hline Stroke & STROKE & 5,176 & $\begin{array}{l}\text { Lippincott Williams } \\
\text { \& Wilkins }\end{array}$ & V Hachinski, MD & $\begin{array}{l}\text { Am Heart (Stroke) } \\
\text { Assoc }\end{array}$ & $\begin{array}{l}\text { http://stroke.ahajournals.org/ } \\
\text { contents-by-date.2001.shtml } \\
\text { (Volltext) }\end{array}$ \\
\hline Pain & PAIN & 4,829 & Elsevier & $\begin{array}{l}\text { R Dubner, Balti- } \\
\text { more }\end{array}$ & $\begin{array}{l}\text { Internat Assoc for } \\
\text { the Study of Pain } \\
\text { (IASS) }\end{array}$ & $\begin{array}{l}\text { www.sciencedirect.com/ } \\
\text { science/journal/03043959 } \\
\text { (Volltext) }\end{array}$ \\
\hline $\begin{array}{l}\text { Neuromuscular } \\
\text { Disorders }\end{array}$ & $\begin{array}{l}\text { NEUROMUSCULAR } \\
\text { DISORD }\end{array}$ & 2,587 & $\begin{array}{l}\text { Pergamon-Elsevier } \\
\text { Science }\end{array}$ & $\begin{array}{l}\text { V Dubowitz, Dept } \\
\text { of Paed, ICSM Lon- } \\
\text { don }\end{array}$ & World Muscle Soc & $\begin{array}{l}\text { www.sciencedirect.com/ } \\
\text { science/journal/09608966 } \\
\text { (Volltext) }\end{array}$ \\
\hline Movement Disorders & $\begin{array}{l}\text { MOVEMENT DIS- } \\
\text { ORD }\end{array}$ & $\begin{array}{l}2,561 \\
(2001)\end{array}$ & Wiley Interscience & $\begin{array}{l}\text { AE Lang, Toronto } \\
\text { Western Hosp }\end{array}$ & Movement Dis Soc & $\begin{array}{l}\text { www.interscience.wiley.com/ } \\
\text { jpages/0885-3185/ }\end{array}$ \\
\hline $\begin{array}{l}\text { Journal of Pain and } \\
\text { Symptom Manage- } \\
\text { ment }\end{array}$ & $\begin{array}{l}\text { J PAIN SYMPTOM } \\
\text { MANAG }\end{array}$ & 2,466 & Elsevier & $\begin{array}{l}\text { R Portenoy, MD, } \\
\text { New York }\end{array}$ & $\begin{array}{l}\text { U.S. Cancer Pain } \\
\text { Relief Committee }\end{array}$ & $\begin{array}{l}\text { www.sciencedirect.com/ } \\
\text { science/journal/08853924 }\end{array}$ \\
\hline Muscle \& Nerve & MUSCLE NERVE & $\begin{array}{l}2,316 \\
(2001)\end{array}$ & $\begin{array}{l}\text { John Wiley \& Sons } \\
\text { Inc }\end{array}$ & $\begin{array}{l}\text { MJ Aminoff, MD, } \\
\text { San Francisco }\end{array}$ & $\begin{array}{l}\text { Am Assoc of Elec- } \\
\text { trodiagn Med }\end{array}$ & $\begin{array}{l}\text { www.interscience.wiley.com/ } \\
\text { jpages/0148-639X/ }\end{array}$ \\
\hline $\begin{array}{l}\text { Clinical Neurophysio- } \\
\text { logy }\end{array}$ & $\begin{array}{l}\text { CLIN NEUROPHY- } \\
\text { SIOL }\end{array}$ & 2,120 & Elsevier & $\begin{array}{l}\text { M Hallett, Bethes- } \\
\text { da }\end{array}$ & $\begin{array}{l}\text { International Fede- } \\
\text { ration of Clinical } \\
\text { Neurophysiology }\end{array}$ & $\begin{array}{l}\text { http://www.sciencedi- } \\
\text { rect.com/science/jour- } \\
\text { nal/13882457 (Volltext) }\end{array}$ \\
\hline Clinical Journal of Pain & CLIN J PAIN & 1,939 & $\begin{array}{l}\text { Lippincott Williams } \\
\& \text { Wilkins }\end{array}$ & DC Turk PhD & & $\begin{array}{l}\text { www.clinicalpain.com/pt/re/ } \\
\text { clnjpain/home.htm }\end{array}$ \\
\hline $\begin{array}{l}\text { Journal of Electromy- } \\
\text { ography \& Kinesiology }\end{array}$ & $\begin{array}{l}\text { J ELECTROMYOGR } \\
\text { KINES }\end{array}$ & 1,425 & $\begin{array}{l}\text { Elsevier Science } \\
\text { Amsterdam }\end{array}$ & $\begin{array}{l}\text { M Solomonov, } \\
\text { New Orleans }\end{array}$ & $\begin{array}{l}\text { Int Soc of Electro- } \\
\text { physiol and Kine- } \\
\text { siol }\end{array}$ & $\begin{array}{l}\text { www.sciencedirect.com/ } \\
\text { science/journal/10506411 } \\
\text { (Volltext) }\end{array}$ \\
\hline $\begin{array}{l}\text { European Journal of } \\
\text { Pain }\end{array}$ & $\begin{array}{l}\text { EUR J PAIN-LON- } \\
\text { DON }\end{array}$ & 1,274 & Elsevier & $\begin{array}{l}\text { Prof F Cerbero, } \\
\text { Madrid }\end{array}$ & $\begin{array}{l}\text { Eur Chapt of the Int } \\
\text { Assoc for the Study } \\
\text { of Pain }\end{array}$ & $\begin{array}{l}\text { www.harcourt-international. } \\
\text { com/journals/eujp/ }\end{array}$ \\
\hline Brain Injury & BRAIN INJURY & 1,043 & Taylor \& Francis & $\begin{array}{l}\text { J Kreutzer, Med } \\
\text { Coll of Virginia }\end{array}$ & $\begin{array}{l}\text { Int Brain Injury As- } \\
\text { soc/Eur Brain Injury } \\
\text { Soc }\end{array}$ & $\begin{array}{l}\text { www.tandf.co.uk/journals/tf/ } \\
\text { 02699052.html }\end{array}$ \\
\hline $\begin{array}{l}\text { Human Movement } \\
\text { Science }\end{array}$ & $\begin{array}{l}\text { HUM MOVEMENT } \\
\text { SCI }\end{array}$ & 0,987 & Elsevier & $\begin{array}{l}\text { P Beek/P van Wie- } \\
\text { ringen Amsterdam }\end{array}$ & & $\begin{array}{l}\text { www.sciencedirect.com/ } \\
\text { science/journal/01679457 } \\
\text { (Volltext) }\end{array}$ \\
\hline Nervenarzt, Der & NERVENARZT & 0,886 & Springer Verlag & $\begin{array}{l}\text { T Brandt, München } \\
\text { H-J Möller, Mün- } \\
\text { chen }\end{array}$ & $\begin{array}{l}\text { D Ges für Psychiat- } \\
\text { rie, Psychoth und } \\
\text { Nervenheilk }\end{array}$ & $\begin{array}{l}\text { http://link.springer.de/link/ } \\
\text { service/journals/00115/ }\end{array}$ \\
\hline $\begin{array}{l}\text { Journal of Musculo- } \\
\text { skeletal Pain }\end{array}$ & $\begin{array}{l}\text { J MUSCULOSCELET } \\
\text { PAIN }\end{array}$ & 0,621 & Haworth Press Inc. & $\begin{array}{l}\text { J Russell, MD, Univ } \\
\text { of Texas, San Anto- } \\
\text { nio }\end{array}$ & Int MYOPAIN Soc & $\begin{array}{l}\text { www.haworthpressinc.com/ } \\
\text { web/JMP/ }\end{array}$ \\
\hline Schmerz & SCHMERZ & 0,602 & $\begin{array}{l}\text { Springer Berlin } \\
\text { Heidelberg }\end{array}$ & M Zenz, Bochum & $\begin{array}{l}\text { D Ges zum Studi- } \\
\text { um des Schmerzes }\end{array}$ & $\begin{array}{l}\text { http://link.springer-ny.com/ } \\
\text { link/service/journals/ } \\
\text { 00482/index.htm }\end{array}$ \\
\hline \multicolumn{7}{|l|}{ Sportmedizin, Exercise } \\
\hline $\begin{array}{l}\text { Journal of Applied } \\
\text { Physiology }\end{array}$ & J APPL PHYSIOL & 2,720 & Am Physiol Soc & GC Sieck & Am Physiol Soc & http://jap.physiology.org/ \\
\hline $\begin{array}{l}\text { Medicine and Science } \\
\text { in Sports and Exercise }\end{array}$ & $\begin{array}{l}\text { MED SCI SPORT } \\
\text { EXER }\end{array}$ & 2,600 & $\begin{array}{l}\text { Lippincott Williams } \\
\& \text { Wilkins }\end{array}$ & $\begin{array}{l}\text { KB Pandolf, Ph. D., } \\
\text { MPH, U.S. Army } \\
\text { Res }\end{array}$ & $\begin{array}{l}\text { Am Coll of Sports } \\
\text { Med }\end{array}$ & www.ms-se.com \\
\hline Sports Medicine & SPORTS MED & 2,281 & $\begin{array}{l}\text { Adis International } \\
\text { Limited }\end{array}$ & & & $\begin{array}{l}\text { www.adis.com/page.asp? } \\
\text { objectID=99 }\end{array}$ \\
\hline $\begin{array}{l}\text { The American Journal } \\
\text { of Sports Medicine }\end{array}$ & AM J SPORT MED & 2,270 & SAGE Publications & B Reider, Chicago & $\begin{array}{l}\text { Am Orthop Soc for } \\
\text { Sports Med }\end{array}$ & $\begin{array}{l}\text { www.sagepub.com/journal. } \\
\text { aspx?pid=9960 }\end{array}$ \\
\hline Gait and Posture & GAIT POSTURE & 1,753 & Elsevier & $\begin{array}{l}\text { TF Novacheck, } \\
\text { Minnesota, }\end{array}$ & $\begin{array}{l}\text { Gait and Clin Mov } \\
\text { Anal Soc }\end{array}$ & $\begin{array}{l}\text { www.sciencedirect.com/ } \\
\text { science/journal/09666362 } \\
\text { (Volltext) }\end{array}$ \\
\hline $\begin{array}{l}\text { Clinical Journal of Sport } \\
\text { Medicine }\end{array}$ & CLIN J SPORT MED & 1,686 & $\begin{array}{l}\text { Lippincott Williams } \\
\& \text { Wilkins }\end{array}$ & $\begin{array}{l}\text { W Meeuwisse MD, } \\
\text { PhD, Calgary, Ca- } \\
\text { nada }\end{array}$ & $\begin{array}{l}\text { Am Med Soc for } \\
\text { Sports Med }\end{array}$ & www.cjsportmed.com \\
\hline $\begin{array}{l}\text { European Journal of } \\
\text { Applied Physiology }\end{array}$ & $\begin{array}{l}\text { EUR J APPL PHYS- } \\
\text { IOL }\end{array}$ & 1,417 & $\begin{array}{l}\text { Springer Verlag. } \\
\text { Heidelberg }\end{array}$ & $\begin{array}{l}\text { PE Prampero, } \\
\text { Udine }\end{array}$ & & $\begin{array}{l}\text { www.springerlink.com/ } \\
\text { link.asp?id=100513 }\end{array}$ \\
\hline $\begin{array}{l}\text { International Journal } \\
\text { of Sports Medicine }\end{array}$ & INT J SPORTS MED & 1,348 & Thieme & $\begin{array}{l}\text { MTE Hopman, } \\
\text { Nijmegen (NL) }\end{array}$ & & www.thieme.de/sportsmed/ \\
\hline $\begin{array}{l}\text { British Journal of Sports } \\
\text { Medicine }\end{array}$ & BRIT J SPORT MED & 1,202 & $\begin{array}{l}\text { BMJ Publishing } \\
\text { Group Ltd }\end{array}$ & $\begin{array}{l}\text { P McCrory, Victo- } \\
\text { ria, Australia }\end{array}$ & $\begin{array}{l}\text { Br Assoc of Sport } \\
\text { and Exerc Med }\end{array}$ & http://bjsm.bmjjournals.com \\
\hline Adapted Physical & ADAPT PHYS ACT & 1,200 & Human Kinetics & DL Porretta, Ohio & Int Fed of Adapted & www.humankinetics.com/ \\
\hline Activity Quarterly & $\mathrm{Q}$ & & Inc. & State Univ & Phys Act & $\begin{array}{l}\text { products/journals/journal. } \\
\text { cfm?id=APAQ }\end{array}$ \\
\hline $\begin{array}{l}\text { Scandinavian Journal of } \\
\text { Medicine and Science } \\
\text { in Sports }\end{array}$ & $\begin{array}{l}\text { SCAND J MED SCI } \\
\text { SPOR }\end{array}$ & 1,117 & Blackwell & $\begin{array}{l}\text { M Kjaer, Copen- } \\
\text { hagen }\end{array}$ & & $\begin{array}{l}\text { www.blackwellpublishing. } \\
\text { com/journal.asp?ref=0905- } \\
7188\end{array}$ \\
\hline
\end{tabular}


Tab. 2 Fortsetzung

\begin{tabular}{|c|c|c|c|c|c|c|}
\hline $\begin{array}{l}\text { Themenschwerpunkt; } \\
\text { Full Title }\end{array}$ & Kurztitel/Short Title & IF 2002 & Verlag & Herausgeber/Editor & Organ/Society & Website \\
\hline Clinical Biomechanics & CLIN BIOMECH & 0,996 & $\begin{array}{l}\text { Elsevier Science } \\
\text { Amsterdam }\end{array}$ & $\begin{array}{l}\text { K Burton, Univ of } \\
\text { Huddersfield }\end{array}$ & $\begin{array}{l}\text { Am Soc of Bio- } \\
\text { mech/Intern Soc } \\
\text { of Biomech }\end{array}$ & $\begin{array}{l}\text { www.sciencedirect.com/sci- } \\
\text { ence/journal/02680033 (Voll- } \\
\text { text) }\end{array}$ \\
\hline $\begin{array}{l}\text { Journal of Strength and } \\
\text { Conditioning Research }\end{array}$ & $\begin{array}{l}\text { J STRENGTH COND } \\
\text { RES }\end{array}$ & 0,762 & Allen Press Inc & $\begin{array}{l}\text { WJ Kraemer, PhD, } \\
\text { CSCS Ball State } \\
\text { Univ }\end{array}$ & $\begin{array}{l}\text { Nat Strength \& } \\
\text { Condit Assoc }\end{array}$ & $\begin{array}{l}\text { http://nsca.allenpress.com/ } \\
\text { nscaonline/?request=index- } \\
\text { html }\end{array}$ \\
\hline $\begin{array}{l}\text { Sportverletzung - } \\
\text { Sportschaden }\end{array}$ & $\begin{array}{l}\text { SPORTVERLETZ } \\
\text { SPORTSC }\end{array}$ & 0,295 & Thieme & $\begin{array}{l}\text { H-P Scharf, Mann- } \\
\text { heim }\end{array}$ & & www.thieme.de/sport/ \\
\hline $\begin{array}{l}\text { Deutsche Zeitschrift für } \\
\text { Sportmedizin }\end{array}$ & Deut Z Sportmed & $\begin{array}{l}\text { kein IF } \\
0\end{array}$ & $\begin{array}{l}\text { V. zur Förderung } \\
\text { Sportmed, Hanno- } \\
\text { ver }\end{array}$ & $\begin{array}{l}\text { JM Steinacker, Re- } \\
\text { hab Med Univ Ulm }\end{array}$ & $\begin{array}{l}\text { D Gesellschaft für } \\
\text { Sportmed und Präv }\end{array}$ & $\begin{array}{l}\text { www.zeitschrift-sportmedi- } \\
\text { zin.de/ }\end{array}$ \\
\hline \multicolumn{7}{|c|}{ Geriatrie, Gerontologie, Aging } \\
\hline $\begin{array}{l}\text { Journal of the American } \\
\text { Geriatrics Society }\end{array}$ & J AM GERIATR SOC & 3,092 & $\begin{array}{l}\text { Lippincott Williams } \\
\& \text { Wilkins }\end{array}$ & TT Yoshikawa, MD & Am Geriatr Soc & $\begin{array}{l}\text { www.blackwellscience.com/ } \\
\text { journals/geriatrics/ }\end{array}$ \\
\hline Age and Ageing & AGE AGEING & 1,600 & $\begin{array}{l}\text { Oxford University } \\
\text { Press }\end{array}$ & GP Mulley & $\mathrm{Br}$ Geriatr Soc & $\begin{array}{l}\text { http://ageing.oupjournals. } \\
\text { org/contents-by-date.2000. } \\
\text { shtml (Volltext) }\end{array}$ \\
\hline $\begin{array}{l}\text { Age, Journal of the } \\
\text { American Aging Asso- } \\
\text { ciation }\end{array}$ & $\begin{array}{l}\text { J AM AGING ASSO- } \\
\text { CAGE }\end{array}$ & 1,486 & $\begin{array}{l}\text { Sally Balin Medical } \\
\text { Center Media PA }\end{array}$ & & Am Aging Assoc & www.americanaging.org/jaaa \\
\hline Gerontology & GERONTOLOGY & 1,480 & Karger Basel & $\begin{array}{l}\text { W Meier-Ruge, Ba- } \\
\text { sel }\end{array}$ & $\begin{array}{l}\text { Int Assoc of Geron- } \\
\text { tol (IAG) }\end{array}$ & $\begin{array}{l}\text { www.karger.ch/journals/ger/ } \\
\text { ger_jh.htm }\end{array}$ \\
\hline $\begin{array}{l}\text { Journal of Aging and } \\
\text { Physical Activity }\end{array}$ & $\begin{array}{l}\text { J AGING PHYS } \\
\text { ACTIV }\end{array}$ & 0,742 & $\begin{array}{l}\text { Human Kinetics } \\
\text { Publications Inc. }\end{array}$ & $\begin{array}{l}\text { WJ Chodzko-Zajko, } \\
\text { PhD, Univ of Illinois }\end{array}$ & $\begin{array}{l}\text { Eur Group for Res } \\
\text { into Elderly }\end{array}$ & $\begin{array}{l}\text { www.humankinetics.com/ } \\
\text { products/journals/journal.cfm } \\
\text { ?id=JAPA }\end{array}$ \\
\hline $\begin{array}{l}\text { Journal of Aging \& } \\
\text { Health }\end{array}$ & J AGING HEALTH & 0,680 & Sage Publ Inc & $\begin{array}{l}\text { KS Markides Univ } \\
\text { of Texas }\end{array}$ & & $\begin{array}{l}\text { www.sagepub.co.uk/journals/ } \\
\text { details/j0100.html }\end{array}$ \\
\hline $\begin{array}{l}\text { Zeitschrift für Geronto- } \\
\text { logie und Geriatrie }\end{array}$ & $\begin{array}{l}\text { Z GERONTOL GER- } \\
\text { IATR }\end{array}$ & 0,441 & $\begin{array}{l}\text { Dr. Dietrich Stein- } \\
\text { kopff Verlag Darm- } \\
\text { stadt }\end{array}$ & $\begin{array}{l}\text { RD Hirsch, Rhein. } \\
\text { Kliniken, Bonn }\end{array}$ & $\begin{array}{l}\text { D Ges für Gerontol } \\
\text { und Geriatr }\end{array}$ & $\begin{array}{l}\text { http://link.springer.de/link/ } \\
\text { service/journals/00391/ }\end{array}$ \\
\hline \multicolumn{7}{|c|}{ Epidemiologie, Public Health } \\
\hline $\begin{array}{l}\text { American Journal of } \\
\text { Epidemiology }\end{array}$ & AM J EPIDEMIOL & 4,189 & $\begin{array}{l}\text { Oxford Univ Press } \\
\text { Inc }\end{array}$ & & $\begin{array}{l}\text { Soc for Epidemiol } \\
\text { Res }\end{array}$ & $\begin{array}{l}\text { www.jhsph.edu/Publications/ } \\
\text { JEPI/ }\end{array}$ \\
\hline Epidemiology & EPIDEMIOLOGY & 3,962 & $\begin{array}{l}\text { Lippincott Williams } \\
\& \text { Wilkins }\end{array}$ & AJ Wilcox & $\begin{array}{l}\text { Int Soc for Environ } \\
\text { Epidemiol }\end{array}$ & www.epidem.com/ \\
\hline $\begin{array}{l}\text { American Journal of } \\
\text { Public Health }\end{array}$ & $\begin{array}{l}\text { AM J PUBLIC } \\
\text { HEALTH }\end{array}$ & 3,279 & $\begin{array}{l}\text { American Public } \\
\text { Health Association }\end{array}$ & $\begin{array}{l}\text { MN Akhter, MD, } \\
\text { MPH }\end{array}$ & $\begin{array}{l}\text { Am Public Health } \\
\text { Assoc }\end{array}$ & www.ajph.org \\
\hline Medical Care & MED CARE & 3,228 & $\begin{array}{l}\text { Lippincott Williams } \\
\& \text { Wilkins }\end{array}$ & $\begin{array}{l}\text { M Weinberger, } \\
\text { PHD Univ of North } \\
\text { Carolina }\end{array}$ & $\begin{array}{l}\text { Med Care Sect- Am } \\
\text { Public Health As- } \\
\text { soc }\end{array}$ & www.lww-medicalcare.com/ \\
\hline $\begin{array}{l}\text { American Journal of } \\
\text { Preventive Medicine }\end{array}$ & AM J PREV MED & 2,630 & Elsevier & $\begin{array}{l}\text { K Patrick, MD, MS, } \\
\text { San Diego, CA }\end{array}$ & $\begin{array}{l}\text { Am College of Pre- } \\
\text { vent. Med./ }\end{array}$ & $\begin{array}{l}\text { www.elsevier.com/locate/ } \\
\text { amepre }\end{array}$ \\
\hline $\begin{array}{l}\text { Health Service Re- } \\
\text { search Journal }\end{array}$ & HEALTH SERV RES & 2,338 & $\begin{array}{l}\text { Health Res \& Educ } \\
\text { Trust }\end{array}$ & M Pittman Dr PH & $\begin{array}{l}\text { Assoc for Health } \\
\text { Serv Res }\end{array}$ & www.hsr.org/ \\
\hline $\begin{array}{l}\text { Journal of Clinical Epi- } \\
\text { demiology }\end{array}$ & J CLIN EPIDEMIOL & 2,223 & Elsevier & $\begin{array}{l}\text { AR Feinstein, Yale } \\
\text { Univ New Haven, } \\
\text { CT }\end{array}$ & & $\begin{array}{l}\text { www.sciencedirect.com/ } \\
\text { science/journal/08954356 } \\
\text { (Volltext) }\end{array}$ \\
\hline Annals of Epidemiology & ANN EPIDEMIOL & 2,214 & Elsevier & $\begin{array}{l}\text { R Rothenberg, } \\
\text { Emory Univ }\end{array}$ & $\begin{array}{l}\text { Am Coll of Epide- } \\
\text { miol }\end{array}$ & $\begin{array}{l}\text { www.sciencedirect.com/ } \\
\text { science/journal/01676296 } \\
\text { (Volltext) }\end{array}$ \\
\hline Public Health Reports & $\begin{array}{l}\text { PUBLIC HEALTH } \\
\text { REP }\end{array}$ & 0,846 & $\begin{array}{l}\text { Oxford University } \\
\text { Press }\end{array}$ & R Rinsky, Boston & & http://phr.oupjournals.org/ \\
\hline $\begin{array}{l}\text { Scandinavian Journal of } \\
\text { Public Health }\end{array}$ & $\begin{array}{l}\text { SCAND J PUBLIC } \\
\text { HEALTH }\end{array}$ & 0,769 & Taylor \& Francis & $\begin{array}{l}\text { S Wall, Umeå, } \\
\text { Sweden }\end{array}$ & & $\begin{array}{l}\text { www.tandf.co.uk/journals/tfs/ } \\
\text { 14034948.html }\end{array}$ \\
\hline $\begin{array}{l}\text { Journal of Public Health } \\
\text { Medicine }\end{array}$ & $\begin{array}{l}\text { J PUBLIC HEALTH } \\
\text { MED }\end{array}$ & 0,761 & $\begin{array}{l}\text { Oxford University } \\
\text { Press Journals }\end{array}$ & $\begin{array}{l}\text { EG Jessop, Cam- } \\
\text { berley, UK }\end{array}$ & $\begin{array}{l}\text { Fac of Public } \\
\text { Health Med, UK }\end{array}$ & $\begin{array}{l}\text { http://jpubhealth.oupjour } \\
\text { nals.org/contents-by-date.0. } \\
\text { shtml }\end{array}$ \\
\hline Public Health & PUBLIC HEALTH & 0,674 & Elsevier & F Sim, P Mackie & $\begin{array}{l}\text { R Inst for Public } \\
\text { Health }\end{array}$ & $\begin{array}{l}\text { http://intl.elsevierhealth.com/ } \\
\text { journals/pubh/Default. cfm }\end{array}$ \\
\hline
\end{tabular}


Autoren unterlagen. Sie wurde ergänzt durch die Abstimmung mit dem Vorstand der DGPMR sowie mit weiteren Fachkollegen im deutschsprachigen Raum. Bei einigen Zeitschriften waren Doppelnennungen bei den entsprechenden Themengebieten erforderlich.

\section{Schlussbemerkung}

Die in Tab. 2 aufgeführte Auswahlliste zeigt eine bemerkenswerte Vielfalt an fachrelevanten Zeitschrifttiteln im Bereich der PMR und unterstreicht damit die Bedeutung und internationale Repräsentanz unseres Fachgebietes. Sie ist mit rund 130 Titeln zwar recht umfangreich, kann aber, wie auch die um mehrere tausend Titel größere Gesamtliste, selbstverständlich keinen Anspruch auf Vollständigkeit erheben. Wir gehen jedoch davon aus, dass die meisten PMR-relevanten Journals und die wichtigsten fachverwandten Bereiche erfasst wurden.

Die Tab. 2 wird künftig auch auf der Website der DGPMR zur Verfügung gestellt (www.dgpmr.de). Hierdurch wird es möglich, per
Mausklick unmittelbar auf die Website jedes Journals zu gelangen und die Titel, Abstracts und z.T. auch die Volltexte der wissenschaftlichen Arbeiten einzusehen. Alle Abonnenten haben darüber hinaus die Möglichkeit, sich über die Website dieser Zeitschrift (www.thieme.de/physmed/) einzuloggen, diesen Beitrag als PDF-Datei herunterzuladen und in Tab. 2 die Websites der erwünschten Journals anzuklicken. Eine regelmäßige Aktualisierung der Internetversion der hier vorgestellten Liste ist notwendig und vorgesehen. Änderungsvorschläge werden von den Autoren dankbar entgegengenommen.

\section{Literatur}

${ }^{1}$ Journal Citation Report (JCR). Philadelphia (USA): Institute for Scientific Information (ISI), http://www.isiknowledge.com

${ }^{2}$ Schreiber TU. Zeitschrift, Impact-Factor und Chancen des Fachgebiets. Phys Med Rehab Kuror 2002; 12: 257-259

${ }^{3}$ Bowman B. Impact Factor. Informationsvermittlungsstelle für die Forschungseinrichtungen der Bio.-Med. Sektion der Max-Planck-Gesellschaft 1998, http://www.biochem.mpg.de/iv/impact.html 